\title{
Atti Sociali
}

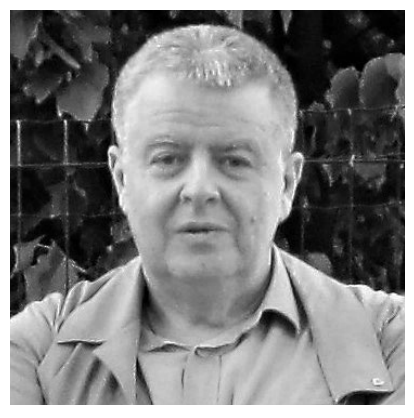

\section{Sergio Riese (1945-2020)}

Sergio Gondrano Riese nacque a Genova Pegli il 12 aprile 1945. Il padre era un militare tedesco che alla fine della guerra ritornò in Germania e morì pochi anni dopo. Riese visse tutta la vita con sua madre, prima per qualche anno in Brasile e poi, al rientro in Italia, a Genova Nervi, zona residenziale del levante cittadino; dopo il servizio militare, svolto in Marina, si impiegò in una compagnia di assicurazioni. Nel 1992 la famiglia lasciò Nervi e acquistò un appartamento più centrale, in Corso Sardegna. Dopo il suo pensionamento (2003) e il decesso di sua madre (2011) anche le condizioni di salute di Riese cominciarono lentamente a peggiorare. Ai problemi legati all'obesità e al diabete si aggiunsero quelli circolatori, tanto che nel 2015 subì un infarto e gli furono applicati 3 bypass. Pur con alcune limitazioni riprese le sue attività, anche se negli ultimi tempi aveva difficoltà a muoversi. Il 3 giugno 2020 un nuovo infarto lo colse per strada, nei pressi della sua abitazione, e purtroppo non gli lasciò scampo. La notizia della sua scomparsa rischiò di passare sotto silenzio, e tale in effetti fu per alcuni giorni, in quanto Riese viveva da solo e non aveva nessun parente.

L'interesse naturalistico era sorto in lui entrando nell'organizzazione scoutistica, alla quale rimase sempre molto legato; per identificare gli Insetti che incominciava a raccogliere si iscrisse nel 1964 al Gruppo Entomologico Ligure e nel 1971 alla nostra Società. Inizialmente si occupò di tutti i Coleotteri, con una propensione per i Carabidi, ma nei primi anni Settanta decise di dedicarsi esclusivamente agli Elateridi, su suggerimento di Giovanni Binaghi, ben noto esperto della famiglia.

Dopo essersi impratichito nella fauna italiana decise di affrontare quella mondiale e da quel momento iniziò un'intensissima attività di acquisizione di materiali. Personalmente non effettuò mai campagne di ricerche in nazioni extraeuropee, ma nel corso degli anni prese contatti in tutto il mondo con ogni sorta di raccoglitore, venditore, mercante o appassionato, intessendo una fitta rete di rapporti internazionali ed effettuando scambi, ma soprattutto acquistando migliaia e migliaia di esemplari.

Numerose furono le specie nuove rinvenute nei lotti che riceveva dai suoi corrispondenti, che egli però descrisse solo in minima parte, preferendo inviare in esame i suoi esemplari, con estrema generosità, ai colleghi che avevano studi in corso su un dato genere o su una particolare regione. Giuseppe Platia, Rainer Schimmel e Claude Girard sono coloro che hanno maggiormente goduto della sua liberalità, ma non c'è specialista mondiale di Elateridi che non sia stato in contatto con lui, a cui sono state dedicate 24 specie nuove oltre ad un genere (Rieseulus). Da parte sua pubblicò 20 contributi ( 1 postumo) in massima parte sul nostro Bollettino, descrivendo 33 specie, soprattutto neotropicali.

Nel dicembre 2017, anticipando una volontà comunicatami già da anni, donò al Museo Civico di Storia Naturale "G. Doria " di Genova la sua collezione di Elateridae, collocata in 432 scatole piccole, comprendente almeno 45.000 esemplari determinati e 4.878 specie (quindi circa la metà di quelle ad oggi note), 590 delle quali rappresentate da 1.500 esemplari tipici. Ad essa si aggiungono le 70 scatole di materiale indeterminato, i tanti duplicati non preparati e la biblioteca specializzata.

Sergio Riese non passava inosservato, sia per la sua corporatura che per la sua personalità; profondamente religioso, due volte all' anno si recava in pellegrinaggio a Lourdes per accompagnare i malati, era donatore di sangue e sosteneva associazioni benefiche e ambientali; era inoltre appassionatissimo di fumetti, di manga giapponesi e di fantascienza.

Aveva un forte senso dell'amicizia, che però esercitava anche con lunghi periodi di totale silenzio a cui ci eravamo ormai tutti abituati e che interrompeva solo in occasione di qualche evento particolare. Nell'ambito della nostra Società è stato eletto continuativamente Revisore dei Conti, sia ordinario che supplente, a partire dal 1994 e sino ad oggi. 
Quanto ha radunato nella sua vita e poi generosamente messo a disposizione degli altri resterà un'indispensabile fonte di dati e di confronti per tutti i futuri elateridologi.

Roberto Poggi

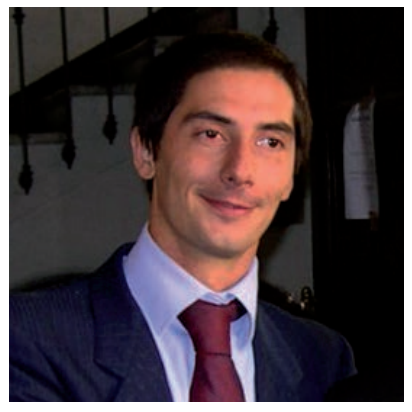

\section{Andrea Sabbadini (1970-2020)}

Il 30 luglio 2020, in un tragico incidente in mare in Sardegna, è deceduto Andrea Sabbadini. Nato a Milano il 30 settembre 1970, Andrea rivelò un talento precocissimo per l'entomologia. Non a caso è stato forse il più giovane tra tutti i nuovi soci della SEI, di cui ha fatto parte dal 1982 al 2014. Ancora ragazzino iniziò a frequentare il Museo di Storia Naturale di Milano, assieme a suoi compagni di scuola, con scatole e scatole di coleotteri di cui voleva conoscere tutti $i$ nomi. Fece in tempo a conoscere un animatore impareggiabile dell'ambiente entomologico milanese come Italo Bucciarelli, ma soprattutto trovò in Carlo Pesarini, che era da poco entrato in Museo come conservatore per gli invertebrati, una guida e un punto di riferimento che si rivelarono fondamentali non solo per la sua maturazione come entomologo ma anche sul piano umano. Con altri giovanissimi, quali i fratelli Pierpaolo e Ivan Rapuzzi, formò un gruppo affiatato di neofiti con cui condivideva la passione per le cacce entomologiche e il piacere di stare insieme, ciò ancora prima di mettere a fuoco quali fossero gli obiettivi, sul piano scientifico, delle proprie investigazioni.

Una volta diplomatosi al Liceo Scientifico Tumminelli di Milano, nel 1991 Andrea venne assunto dietro concorso al Museo di Storia Naturale con mansioni di tecnico, dove lavorò affiancato a mio fratello Carlo fino al pensionamento di questi, avvenuto nel marzo del 2013. Fu grazie ad Andrea, assieme al quale si appassionò allo studio dei Cerambycidae, che Carlo conobbe una sorta di seconda giovinezza entomologica: insieme condussero innumerevoli campagne di raccolta, specie in Grecia e Turchia, e pubblicarono come coautori ben 27 contributi in cui descrissero 108 taxa nuovi per la scienza. Da parte sua, Andrea ha inoltre tradotto dall'inglese e curato l'edizione italiana di alcuni noti e diffusi manuali naturalistici.

Andrea Sabbadini era, sia da ragazzo che poi come uomo, una personalità ricchissima di interessi, di umanità schietta e profondamente amante della natura e dell'avventura: escursionista esperto, rocciatore, tra le sue passioni c'erano pure la mountain bike e il kayak; ma amava altrettanto il mare e lo snorkelling. In tutto ciò, come nei viaggi in capo al mondo (Australia, Patagonia, Islanda), era sempre assieme alla moglie Stefania e alla piccola Arianna, che oggi ha quindici anni. Ad entrambe e a tutti i congiunti vanno le più sentite e commosse condoglianze della Società.

Fausto Pesarini

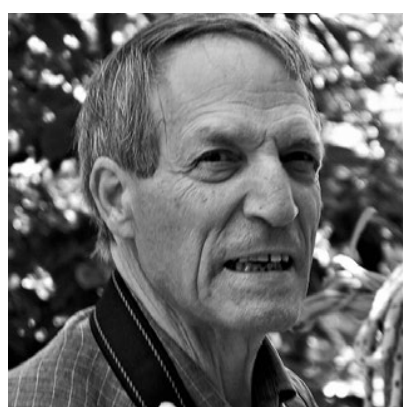

\section{Giovanni Salamanna (1938-2020)}

Giovanni Salamanna era nato a Gallipoli (in provincia di Lecce) il 17 gennaio 1938; trasferitosi poi con la famiglia a Mesagne (in provincia di Brindisi), dopo il Liceo classico si iscrisse all'Università di Bari, dove si laureò in Scienze Naturali, iniziando poi nel 1964 la sua carriera accademica nell'Istituto di Zoologia ed Anatomia Comparata, allora diretto dal Prof. Michele Sarà, studioso di Poriferi ma pure di Ditteri Psicodidi. Indirizzato da Sarà, anche Salamanna incominciò ad interessarsi di questo gruppo di insetti, pur senza trascurare la passione giovanile per i Coleotteri Scarabeoidei oggetto della sua tesi e di cui aveva iniziato a studiare i cromosomi.

Nel 1970, come Assistente Ordinario, si trasferì a Genova seguendo Sarà che vi era giunto l'anno prima per dirigere l'Istituto di Zoologia; qui abbandonò gradatamente gli studi cariologici e si dedicò esclusivamente agli Psicodidi, utilizzando sia i materiali consegnatigli da Sarà (che invece prese ad occuparsi solo di Poriferi e di Biologia marina) sia quelli raccolti da lui stesso e da amici e colleghi in una serie 
di campagne di ricerca. Pubblicò così una trentina di lavori, descrivendo, da solo o in collaborazione, 36 specie nuove, in particolare di Liguria e di Sardegna. Divenuto Professore Associato nel 1981, scelse poi di non partecipare a concorsi di ordinariato per evitare di dover abbandonare Genova, in cui diceva sempre di trovarsi benissimo.

Iniziò in contemporanea ad impegnarsi sempre più intensamente nella didattica, sia per i corsi universitari di cui fu docente (Zoologia, Etologia, Ecoetologia) sia per quelli che tenne gratuitamente per anni nell'ambito del Pro Natura Genova, utilizzando a tale scopo una parte dell'infinita serie di diapositive che andava accumulando grazie alla sua passione per la tecnica fotografica, in cui raggiunse punte di assoluto rilievo nell'ambito della macrofotografia, vincendo anche un premio internazionale.

Alla fine degli anni '80 lo sviluppo dell'informatica costituì per Salamanna un'altra fonte di primario interesse; ben presto infatti egli si dedicò allo sviluppo di programmi per l'archiviazione e la divulgazione giungendo da autodidatta ad imparare ad utilizzare il linguaggio macchina.

La coltivazione di tutte queste passioni (cui si aggiunse per lungo tempo anche quella per i bonsai), unita ad una certa ritrosia nello scrivere, fece sì che Salamanna abbia dato alle stampe molto meno di quello che avrebbe potuto pubblicare e così sono purtroppo rimaste inedite ad esempio le descrizioni delle specie nuove ricevute dall'Himalaya oppure i risultati delle raccolte da lui effettuate in Spagna; in pratica chiuse la sua produzione scientifica col 1996.

A sottrargli tempo per le proprie ricerche era intervenuta anche, nel 1978, l'elezione a Direttore delle Pubblicazioni della nostra Società, cui si era iscritto nel 1963. Salamanna mantenne tale carica fino al 1996, quindi per quasi vent'anni, dedicando a questo compito, spesso ingrato e poco considerato, una gran parte del suo tempo libero, con spirito di vero servizio, impratichendosi talmente nelle pratiche redazionali da arrivare poi ad impaginare da solo il Bollettino e le Memorie.

Dopo il pensionamento, avvenuto nel 2008, Salamanna intraprese un riordino generale della sua ricca collezione di Psicodidi (non Flebotomini), composta da oltre 11.000 vetrini microscopici perfettamente etichettati più vario materiale in alcool, un "unicum" a livello italiano, che ha poi destinato, insieme ai suoi schedari, al Museo Civico di Storia Naturale di Genova, dove è oggi conservata e a disposizione degli studiosi che ne vorranno usufruire.

Salamanna è stato sempre un assiduo frequentatore delle riunioni svolte al sabato nei locali della Biblioteca della S.E.I., prima nella vecchia sede di Corso Magenta e poi in quella nuova di Corso Torino, sempre gentile, aperto e disponibile alla collaborazione con i colleghi. Da un paio d'anni però aveva iniziato a diradare le sue visite, per i primi deboli segnali di quel subdolo e perfido morbo di Alzheimer che lo ha poi accompagnato in modo devastante negli ultimi mesi della vita, chiusasi infine il 6 agosto 2020.

Memore di quanto Gianni ha fatto per tutti noi che abbiamo avuto l'onore di essere considerati suoi amici (e chi scrive lo è stato esattamente per 50 anni), la Società Entomologica Italiana porge alla moglie Carmela, alla figlia Sabrina e a tutti i familiari le più sentite condoglianze.

Roberto Poggi

\section{Assemblea Generale Ordinaria dei Soci del 12 Giugno 2020}

L'Assemblea Generale Ordinaria della Società Entomologica Italiana ha luogo venerdì 12 giugno 2020, alle ore 18, in via telematica tramite piattaforma ZOOM, nell'ambito e a conclusione delle giornate culturali 2020 "Insect Biodiversity and Ecosystem services", organizzate dalla Società stessa e dall'Accademia Nazionale di Entomologia. L'assemblea è stata preceduta da una relazione del Consigliere Avv. Alberto Ballerio dal titolo "La tutela della biodiversità tra mito e realtà: il futuro dell'entomologia professionale ed amatoriale".

Nel corso dell'Assemblea viene discusso il seguente:

\section{ORDINE DEL GIORNO}

1) Convalida dei Soci presentati dal Consiglio Direttivo

2) Comunicazioni della Presidenza

3) Bilancio consuntivo esercizio 2019 e previsioni per il 2020 
4) Relazione dei membri del Consiglio

5) Sviluppo di Entomata

6) Nomina socio onorario

7) Varie ed eventuali

Partecipano 130 soci senza alcuna delega. A presiedere l'Assemblea viene eletto il Presidente della Società, Prof. Francesco Pennacchio.

1) Sono approvati all'unanimità 30 nuovi soci (tutti ordinari).

2) Il Presidente sottolinea l'alta partecipazione e l'intenso coinvolgimento alle giornate culturali appena conclusesi, specialmente da parte di dottorandi e studenti, che hanno dimostrato particolare apprezzamento per l'evento. Ribadisce, quindi, la possibilità di organizzare eventi futuri similari, la cui modalità di organizzazione (in presenza o misti) verrà stabilita a seconda dell'evolversi dell'emergenza sanitaria. La versatilità della piattaforma ZOOM, di cui si è dotata la società ha grandi potenzialità per organizzare simili eventi futuri. Il primo, tra questi, sarà la tavola rotonda "Salute delle piante: una visione One-Health" in collaborazione con l'Accademia Nazionale Italiana di Entomologia, la Società Italiana di Patologia Vegetale e l'Associazione Italiana per la Protezione delle Piante e si terrà il 13 luglio 2020.

A breve, SEI ed ANIE sottoscriveranno con l'Università degli Studi di Napoli Federico II una convenzione per la gestione congiunta di un premio internazionale, dedicato alla memoria di Filippo Silvestri supportato dalle risorse finanziarie derivanti dallo scioglimento della Fondazione Silvestri, costituita negli anni '60, le cui risorse sono state allocate all'Università. La gestione congiunta darà il massimo risalto nazionale e internazionale al premio, che potrà essere indetto a partire dal 2021.

Infine, si ricorda che la scadenza per la presentazione delle domande al Premio Binaghi è stata prorogata dal 30 marzo al 30 settembre p.v..

3) Per via dell'emergenza sanitaria dovuta alla pandemia di COVID-19, che ha comportato il rinvio dell'Assemblea Generale Ordinaria, il bilancio della società è stato approvato, in via eccezionale, dal Consiglio Direttivo in data 22 marzo 2020. Il bilancio 2019 è in regola con gli anni precedenti e non si registrano criticità.

4) Il Direttore delle Pubblicazioni, Dr. Pier Mauro Giachino, riferisce che non vi sono stati problemi per la stampa e la redazione del prossimi numeri del Bollettino nonostante le problematiche inerenti il lockdown. Inoltre, è in fase di preparazione il volume delle Memorie del 2020, dedicato alla memoria del Prof. Augusto Vigna Taglianti, che pubblicherà contributi su invito. Infine, sono già pervenuti lavori per il volume delle Memorie del 2021.

Il Bibliotecario, Dr. Antonio Rey, redige un quadro sulla situazione finanziaria e bibliotecaria. In particolare, per quanto riguarda quest'ultima si sottolinea come al momento la Società mantenga 150 riviste in cambio, a cui se ne aggiunge una decina in abbonamento. Inoltre, per via dell'esiguità delle risorse disponibili si predilige l'acquisto di faune o di volumi di ampio interesse.

5) Il Consigliere Avv. Alberto Ballerio suggerisce di valutare la possibilità di dotare la newsletter Entomata di ISSN al fine di conferire maggiore risalto a questa pubblicazione della Società. Inoltre, sottolinea che una delle criticità è la mancanza di contributi e la scarsa partecipazione da parte dei soci. Dei due numeri annuali di Entomata, uno sarà generalista, mentre il secondo sarà gestito dalla SEI SEA.

6) Il Presidente formalizza la nomina a socio onorario del Prof. Romano Dallai, past president dell'Accademia Nazionale Italiana di Entomologia e scienziato di chiara fama, già proposta durante il consiglio della Società tenutosi a Genova nel dicembre 2019.

7) Il Vicepresidente, Dr. Roberto Poggi, sottolinea come l'assemblea online abbia avuto una partecipazione di gran lunga superiore agli incontri precedenti. Riporta, infine, la mesta notizia del decesso di uno dei revisori dei conti, il Sig. Sergio Riese, deceduto a Genova il 3 giugno 2020. 


\section{Nel corso dell'ultima assemblea generale ordinaria sono stati ammessi i seguenti nuovi soci:}

\section{PER IL 2019}

Dr. Matteo Brunetti, Via Decio Azzolino 62, 00168 Roma (RM)

Prof. Eric ConTI, Strada del Colle 4, 06132 Perugia (PG)

Sig. Maurizio LuPI, Via Zaccaria 2/2, 16156 Genova (GE)

Dr.ssa Giulia MAgoga, Via Luigi Biraghi 15, 20159 Milano (MI) (Coleoptera Chrysomelidae)

Dr. Alessandro Marmugi, Via Maccabelli 15, 16019 Ronco Scrivia (GE)

Dr. Rodolfo OcChIPINTI, Via Vittorio Veneto 32, 97018 Scigli (RG) (Entomologia agraria)

Dr. Luciano TomA, Istituto Superiore di Sanità, Viale Regina Elena 299,00161 Roma (RM) (Ixodida, Diptera Nematocera, Coleoptera Bruchidae)

\section{PER IL 2020}

Dr. Daniele AnCillotTi, Via Orbignanese 64, 51035 Lamporecchio (PT)

Dr.ssa Eleonora BARRA, Via Libertà 336, 80055 Portici (NA) (Interazioni tra insetti ed altri organismi viventi, sistema immunitario degli insetti)

Dr. Andrea Becchimanzi, Piazza Bellini 75, 80138 Napoli (NA)

Dr. Nicola Bodino, via Tripoli 6, 10136 Torino (TO) (Controllo biologico in ambito agricolo, insetti vettori, ecologia acroecosistemi)

Dr. Giacomo Cavaletto, Via Europa 18A, 35020 Sant'Angelo di Piove di Sacco (PD)

Prof. Pierfilippo CerRetTI, Sapienza Università di Roma, Piazzale A. Moro 5, 00185 Roma (RM) (Diptera Oestroidea)

Dr. Marco ColacCI, Via Molise 85, 86021 Boiano (CB)

Dr. Antonino Cusumano, Viale delle Scienze 13, 90128 Palermo (PA) (Interazione insetto-pianta, ecologia chimica controllo biologico, parassitoidi oofagi)

Sig. Marcello De Giosa, Università degli Studi di Bari Di.S.S.P.A., 70125 Bari (BR) (Acarologia ed entomologia agraria)

Dr.ssa Ilaria Di LeLIo, Laboratorio di Entomologia “E. Tremblay”, Via Università 100, 80055 Portici (NA) (Interazione fitofagi ed antagonisti, controllo fitofagi, sistema immunitario degli insetti)

Dr.ssa Giulia GiunTI,Via Madonna di Fatima 10/B, 89124 Reggio Calabria (RC) (Ecologia ed etologia)

Dr.ssa Isabel Martinez SANudo, Via G. Leopardi, 35010 Cadoneghe (PD)

Dr. Antonio MASETTI, Via G. Fanin 42,40127 Bologna (BO) (Lotta biologica conservativa, biodiversità negli agroecosistemi)

Dr. Giacomo OrTIS, Via Monte San Marco 57, 33100 Udine (UD)

Dr. Stefan Cristian Prazaru, Via Sassara 45, 30039 Strà (VE)

Dr. Luca Rossinı, Via degli Olmi 10, 00036 Palestrina (RM) (Modelli matematici di popolazione di insetti di interesse agrario e forestale)

Dr. Michele RossinI, Via Strada del Molino Mosca, 61121 Pesaro (PU) (Tassonomia, sistematica e biogegrafia di Coleoptera Scarabeidae)

Dr. Elia Russo, Via Benedetto Croce 15/B5, 80055 Portici (NA) (Pest control)

Dr. Francesco SACCO, Via Lungotevere Ripa 3/B, 00153 Roma (RM) (Coleoptera Apionidae)

Dr. Giorgio Sperandio, Via Fratelli Bandiera 23, 25122 Brescia (BS) (Strumenti quantitativi per la gestione di insetti invasivi e parassiti delle piante, salute delle api ed apicoltura)

Dr. Giovanni TAMBURINI, Via Fornasari 7, 35133 Padova (PV) (Parassiti delle colture, controllo biologico, impollinazione, ecologia del paesaggio)

Dr. Gennaro VolPE, Via Ignazio Falconeri 37, 80141 Napoli (NA) (Insect pest control)

Dr.ssa Veronica VizZARri, Via Perugia 34/B, 87046 Montalto Uffugo (CS) (strategie di lotta ai parassiti, fitofagi (soprattutto Bactrocera oleae, mosca dell'olivo) e patogeni, dell'olivo)

Dr. Matteo Zugno, Via Scalabrini 55, 22073 Fino Mornasco (CO) 


\section{SOCIETA' ENTOMOLOGICA ITALIANA}

\section{BILANCIO CONSUNTIVO 2019}

\section{STATO PATRIMONIALE AL 31.12.2019}

Patrimonio Sociale

Immobile Sede Biblioteca Sociale (valore catastale riv.)

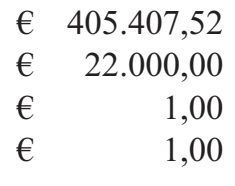

Fondo riserva

Mobili \& Attrezzi (pro memoria)

Biblioteca (pro memoria)

LIQUIDITÀ AL 31.12.2019

Conto corrente bancario

$€ \quad 20.330,80$

Conto corrente postale

$€ \quad 4.031,24$

$€ \quad 24.362,04$

Totale $€ \quad 451.769,56$

II. RENDICONTO DI CASSA AL 31.12.2019

INTROITI

1. Liquidità al 31.12.2018

2. Quote sociali (importi netti)

3. Contributo $5 \%$ ex-IRPEF

4. Contributo ministeriale

5. Rimborsi

\begin{tabular}{rr}
$€$ & $22.686,15$ \\
$€$ & $16.279,50$ \\
$€$ & $4.184,84$ \\
$€$ & $6.408,00$ \\
$€$ & $2.135,78$ \\
\cline { 2 - 2 } Totale $€$ & $51.694,27$
\end{tabular}

ESBORSI

6. Conto economico

$€ \quad 15.898,81$

7. Costo pubblicazioni sociali (stampa, confezione \& spedizione)

$€ \quad 11.433,42$

LIQUIDITÀ FINALE AL 31.12.2019 (come da stato patrimoniale)

8. Accantonamento a fronte rischi

\begin{tabular}{rr} 
Totale $€$ & $27.332,23$ \\
\cline { 2 - 2 }$€$ & $24.362,04$ \\
$€$ & $20.000,00$ \\
\cline { 2 - 2 } Totale $€$ & $4.362,04$
\end{tabular}

RATEI PASSIVI AL 31.12.2019

Spese pubblicazione (a calcolo)

Stampa, confezione e spedizione

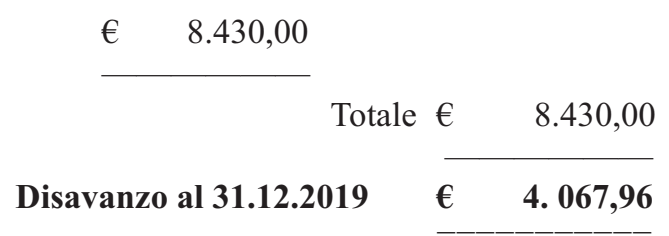




\section{DIMOSTRAZIONE DEL CONTO ECONOMICO 2019}

6.1 - Gestione ordinaria Sede Sociale

6.2 - Biblioteca Sociale

$€ \quad 6.766,11$

6.3 - Fondo minute spese Segreteria

$€ \quad 3.001,41$

6.4 - Fondo minute spese Biblioteca

$€ \quad 300,00$

6.5 - Fondo minute spese Amministrazione

$€ \quad 500,00$

6.6 - Postali e bancarie ordinarie

$€ \quad 200,00$

6.7 - Spese spedizioni

$€ \quad 496,79$

6.8 - Assicurazioni diverse

$€ \quad 808,50$

$€ \quad 640,92$

6.9 - Imposte e tasse

$1.185,08$

6.10 - Contributo congresso internazionale

$€ \quad 2.000,00$

Saldo al 31.12.2019 (come da rendiconto di cassa)

$€ \quad 15.898,81$

\section{BILANCIO PREVENTIVO ANNO 2020}

INTROITI

1. Quote sociali

$€ \quad 16.000,00$

2. Utilizzo fondo di riserva

$€ \quad 20.000,00$

Totale $€ \quad \overline{36.000,00}$

ESBORSI

3. Ratei passivi al 31.12.2019

4. Spese pubblicazione (stampa, confezione, spedizione)

5. Biblioteca sociale

6. Spese generali di gestione, incl. imposte e tasse

\begin{tabular}{rr}
$€$ & $9.000,00$ \\
$€$ & $15.000,00$ \\
$€$ & $3.000,00$ \\
$€$ & $9.000,00$ \\
\cline { 2 - 2 } Totale & $36.000,00$
\end{tabular}

L'Amministratore

Il Bibliotecario

(Giulio Gardini)

(Antonio Rey)

Il Presidente SEI

(Prof. Francesco Pennacchio) 